\title{
Systematic development of a text-driven and a video-driven web-based computer-tailored obesity prevention intervention
}

\author{
Michel Jean Louis Walthouwer ${ }^{1,2^{*}}$, Anke Oenema ${ }^{1,2}$, Katja Soetens $^{1,2}$, Lilian Lechner ${ }^{1,3}$ and Hein De Vries ${ }^{1,2}$
}

\begin{abstract}
Background: This paper describes the systematic development of a text-driven and a video-driven web-based computer-tailored intervention aimed to prevent obesity among normal weight and overweight adults. We hypothesize that the video-driven intervention will be more effective and appealing for individuals with a low level of education.

Methods and Design: The Intervention Mapping protocol was used to develop the interventions, which have exactly the same educational content but differ in the format in which the information is delivered. One intervention is fully text-based, while in the other intervention in addition to text-based feedback, the core messages are provided by means of videos. The aim of the interventions is to prevent weight gain or achieve modest weight loss by making small changes in dietary intake or physical activity. The content of the interventions is based on the I-Change Model and self-regulation theories and includes behavior change methods such as consciousness raising, tailored feedback on behavior and cognitions, goal setting, action and coping planning, and evaluation of goal pursuit. The interventions consist of six sessions. In the first two sessions, participants will set weight and behavioral change goals and form plans for specific actions to achieve the desired goals. In the remaining four sessions, participants' will evaluate their progress toward achievement of the behavioral and weight goals. They will also receive personalized feedback on how to deal with difficulties they may encounter, including the opportunity to make coping plans and the possibility to learn from experiences of others. The efficacy and appreciation of the interventions will be examined by means of a three-group randomized controlled trial using a waiting list control group. Measurements will take place at baseline and six and twelve months after baseline. Primary outcome measures are body mass index, physical activity, and dietary intake.

Discussion: The present paper provides insight into how web-based computer-tailored obesity prevention interventions consisting of self-regulation concepts and text-driven and video-driven messages can be developed systematically. The evaluation of the interventions will provide insight into their efficacy and will result in recommendations for future web-based computer-tailored interventions and the additional value of using video tailoring.
\end{abstract}

Trial registration: NTR3501.

Keywords: Obesity, Physical activity, Diet, Intervention mapping protocol, Computer-tailoring, Weight management, Education level

\footnotetext{
* Correspondence: michel.walthouwer@maastrichtuniversity.nl

'School for Public Health and Primary Care (CAPHRI), Maastricht University,

Maastricht, The Netherlands

${ }^{2}$ Department of Health Promotion, Maastricht University, P.O. Box 616,

Maastricht 6200MD, The Netherlands

Full list of author information is available at the end of the article
} 


\section{Background}

Obesity is a major global health problem [1,2]. The World Health Organization estimated that in 2008 about 502 million adults worldwide were obese [2] and it is expected that this figure will continue to rise in the future [1]. An individual is classified as obese when the Body Mass Index (BMI: weight in kilograms / height in meters ${ }^{2}$ ) is 30 or higher [3]. In the Netherlands, about $12 \%$ of the adult population is obese and it is expected that this proportion will rise to $18 \%$ by 2024 [4]. There is, however, a significant difference in obesity prevalence between individuals with a lower and a higher level of education [5-7]. In the Netherlands, $18.4 \%$ of individuals with a low level of education are obese, while this percentage is only $6.5 \%$ among people with a higher level of education, making the lower educated group an important target group for obesity prevention programs [5]. These high and increasing prevalence rates are worrying, particularly because obesity is associated with a range of health problems, such as type 2 diabetes, cardiovascular diseases, musculoskeletal disorders, psychological disturbance, different types of cancer, and premature death $[8,9]$. Obesity also causes a considerable economic burden, due to health care use, absenteeism, disability payments, and loss of productivity [1,8-10]. Because of the high burden of obesity, its prevention has become a high priority in many international and national public health policies $[4,11]$.

Nevertheless, effective and efficient interventions to prevent obesity that can reach large numbers of people are scarce $[12,13]$. Moreover, these interventions are not always specifically geared to meet the needs of individuals with a low level of education [5,6,14]. Overall, it can be concluded that there is an urgent need for effective interventions to prevent obesity that can reach large numbers of people and that also appeal to and are effective for individuals with a lower level of education.

Ideally, obesity prevention interventions should have the possibility to adapt to an individual's characteristics, because in general each individual wants to manage his/ her body weight in his/her own specific way $[15,16]$. Web-based computer-tailored interventions may fulfill this requirement since these interventions can provide personally adapted messages based on an individual's responses to, for example, an online questionnaire $[17,18]$. In the last decade, attention to web-based computertailored interventions has increased because of their potential advantages such as the ability to reach many individuals in a relatively cheap way [19] and the possibility for participants to use the intervention whenever they prefer [20]. Further, as web-based computer-tailored interventions require minimal human contact, they are also potentially cost-effective [21]. In addition, because the content of a web-based computer-tailored intervention can be adapted to an individual's characteristics, it is suggested that messages are better read, saved, and remembered as compared to non-tailored materials [22]. Several reviews have already reported the promising effects of web-based computer-tailored interventions for modifying dietary and physical activity behavior $[18,23]$ and preventing weight gain [24]. Web-based computertailored interventions may, therefore, be a good innovation to help in targeting the public health problem of obesity.

We developed two versions of a web-based computertailored intervention aimed to prevent obesity among Dutch adults with a healthy weight or limited overweight (BMI between 18.5 and 30). Both interventions have exactly the same educational content, but differ in the format in which the information is delivered. One intervention is fully text-based, without the use of visual elements (text-text intervention), while the other provides the core messages by means of videos with additional text-based information (video-text intervention). Although tailoring is considered to be an effective health education technique, the way messages are communicated may be even more important. We hypothesize that the video-text intervention will be more appealing and effective for individuals with a low level of education. Lower educated individuals have, for example, lower literacy skills, making textual messages less effective for these individuals [25]. Providing messages by means of other communication formats, such as videos, may therefore be a better way to reach these individuals [26]. An advantage of video messages is the fact that these do not require abstract text to be translated into concrete actions [27], which lower educated individuals often find harder to do $[25,28]$. Video messages reduce the cognitive effort needed to process information, which will free resources to process the main message and accordingly lead to a better understanding [29]. Research has already shown that videos can effectively attract attention and stimulate comprehension in lower educated individuals $[26,30,31]$, while another study has shown a media preference in these individuals for video over text [32]. In addition, there is evidence that videos can be effective in improving cognitions such knowledge, attitude, selfefficacy, and intention [33,34] as well as actual behavior $[35,36]$. Hence, video-text interventions may be a promising approach to effectively increase the attention and comprehension of individuals with a low level of education, which will facilitate information processing and ultimately behavior change $[37,38]$.

As the use of a planned approach during intervention development increases the likelihood for an intervention to be effective [39], we used the Intervention Mapping (IM) protocol [40] to develop the two versions of the intervention and the corresponding implementation and evaluation plan. The IM protocol is a framework for effective theory- 
and evidence-based decision making at each step in intervention development, implementation, and evaluation. The protocol consists of six succeeding steps that can be worked through in an iterative manner. The aim of this paper is to describe the systematic development of the two web-based computer-tailored interventions according to the six steps of the IM protocol.

\section{Methods and design}

The interventions were developed by systematically going through the six steps of the IM protocol. In the first step of this protocol, a needs assessment is carried out using the PRECEDE model [41]. The second step provides the foundation for the intervention by specifying in detail who and what will be changed through the intervention. In step three, theory-based methods and practical applications are identified that can be used to achieve the intervention goals. Step four concerns the actual program development, by using all the products of the previous steps; during this step, a pretest of the intervention is conducted as well. In step five, adoption and implementation plans are specified. Finally, in step six, both an effect and process evaluation plan are developed.

\section{Step 1: needs assessment}

Obesity is caused by an energy imbalance, in which energy intake exceeds energy expenditure [42]. Important obesity-related dietary behaviors associated with a high energy intake are consuming energy-dense food (i.e. high in fat, salt, and sugars), drinking sugared drinks and alcohol, and consuming large portions of foods [42]. Energy can be expended by means of physical activity, which can be divided in different sub-behaviors such as active transportation (e.g. cycling to work), daily activities (e.g. cleaning), leisure time activities (e.g. walking), and sports [9]. Hence, physical activity and dietary intake are important targets in the prevention of obesity [9]. By improving these two behaviors the energy balance can be restored, which in turn will prevent weight gain. In line with the fact that individuals with low levels of education have the highest rate of obesity, they also tend to have the highest energy intake [43] and the lowest physical activity levels [44]. This confirms the importance of developing interventions that are appealing and effective for these individuals.

Prevention of obesity is thought to be most successful when focusing on small changes in dietary intake and physical activity, since small changes are easier to initiate and maintain than large changes as strict diets or following an intensive sport schedule [45]. Further, achieving small changes could lead to an increased self-efficacy, which in turn could stimulate people to make additional small changes [45]. This small changes approach assumes that changes of about 100 kilocalories (kcal) per day are sufficient to ensure an energy balance which is in equilibrium and accordingly prevent weight gain [45]. This is equal to about 2,000 more steps of walking per day or 20 more minutes of other physical activities per day. To consume $100 \mathrm{kcal}$ less per day, individuals only have to consume a little less of energy-dense food or substitute food such as a regular soda for a diet soda or a snack for a piece of fruit. These kinds of moderate energy reductions are sufficient to result in sustained decreases in energy intake without increased feelings of hunger [46]. To lose a little weight (about five kilogram in one year), individuals have to make daily changes of $200 \mathrm{kcal}$.

Based on the small changes approach, the primary objective of the two web-based computer-tailored interventions is to realize small changes in physical activity and dietary intake of 100 or $200 \mathrm{kcal}$ per day in order to prevent obesity (i.e. maintain weight or achieve modest weight loss) among Dutch adults with a healthy weight or limited overweight.

\section{Step 2: program objectives}

The goal of step two is to formulate change objectives, which are the most proximal goals of an intervention that help in achieving the behavioral and weight goals. The two components that are needed to formulate change objectives are performance objectives (i.e. subbehaviors within the desired behavior) and their determinants [40].

\section{Performance objectives}

It has been suggested that in particular self-regulation concepts are important to successfully achieve weight management goals, such as identification of a problem, setting goals, making plans, monitoring weight and behavior, and having coping skills $[39,47]$. Evidence has, for example, shown that individuals with adequate selfregulation skills are more successful in changing complex and habitual behaviors such as physical activity and dietary intake as well as in achieving weight maintenance $[48,49]$. The performance objectives (PO's) and accordingly the content of the interventions were, therefore, based on the concepts and phases of self-regulation models. For example, the first PO is individuals decide to prevent weight gain: maintain current weight or lose a little weight'. An overview of all PO's can be found in Additional file 1 (Table S1). It should be noted that although these PO's follow a sequence of action, it is possible to return to previous PO's.

\section{Determinants}

The next step was to identify the most relevant (i.e. important and changeable) determinants of each PO. For this purpose we carried out an extensive literature review on determinant studies. During this process, the 
I-Change Model [37] was used as framework to identify determinants for the pre-motivational, motivational, and post-motivational phase. This model is an integration of various behavior-oriented theories such as the Social Cognitive Theory, Health Belief Model, Theory of Planned Behavior, Transtheoretical Model, and Goal Setting theories. Based on a determinant analysis, the most relevant determinants that need to be targeted in the intervention are knowledge, awareness, risk perception, attitude, social influence, self-efficacy, intention, skills, and action planning (see Additional file 1: Table S1).

\section{Change objectives}

Change objectives (CO's) specify what individuals have to learn in order to accomplish PO's [40]. CO's are created by combining the PO's and determinants. In total, we formulated around 150 CO's. An example of a CO concerning PO 6 (i.e. make coping plans to deal with difficulties) and the determinant knowledge is individuals can explain how to deal with encountered difficulties'. Additional file 1 (Table S1) provides a selection of the most important CO's per PO and determinant.

\section{Step 3: methods and applications}

In the third step, theoretical methods for changing each determinant were selected and translated to practical applications. Methods and applications were retrieved from literature concerning behavior change techniques and study protocols of previous studies [40,50,51]. Additional file 1 (Table S1) provides an overview of the most important theoretical methods and practical applications of the program. When applicable, this table also shows the differences in practical applications between the texttext and video-text intervention. The most important methods and applications are elucidated in more detail below. It should be noted that both interventions have exactly the same educational content in order to allow for a direct comparison between the text-text intervention and the video-text intervention. For the video-text intervention, particularly the practical applications related to PO 1, 2, 7, and 8 are converted into videos.

\section{Providing information}

To increase an individual's knowledge and skills, providing information is included as theoretical method. In line with the parameters of this method (e.g. information must be relevant) [40], the program mainly consists of basic information that individuals are required to have in order to change their behavior successfully. Individuals will, for example, receive information about the small changes approach (PO 2). To prevent that participants have to read something that they already know or are not interested in, they mostly have the possibility to choose which information they want to read. The program, for example, consists of optional in-depth information, such as the amount of kcal in various food products, which can be read by clicking on hyperlinks. Important information, such as information about the small changes approach, is included in the regular sequence of the program, as this information is important for making further steps in the process. In both the video-text as text-text intervention, all information is provided by means of text-based messages.

\section{Consciousness raising and feedback}

Consciousness raising and feedback will be used to increase individuals' awareness about their body weight (PO 1 and 8), physical activity level, and dietary intake (PO 2, 7, and 9). For this purpose, individuals' have to answer various questions about, for example, their body weight and general dietary intake and physical activity pattern. Based on their answers, they will receive tailored feedback about how they can improve their weight and behavior. This feedback will help participants to set appropriate weight and behavior change goals. In the video-text intervention, all these messages will be delivered using videos. In these videos, the feedback is given by actors playing an expert in the field of nutrition and physical activity. Simultaneously with the video message, a table is shown below the videos consisting of very specific information, for example the different food products a participant consumes or a participant's BMI. In the text-text intervention, the same table will be provided only accompanied with text-based messages.

\section{Decisional balance}

To help participants decide which behavior change would suit them best (PO 2), a decisional balance format is included as well. For this purpose, individuals will be asked about their attitudinal, self-efficacy, and social influence beliefs regarding physical activity and dietary intake. Based on their answers, the program will suggest which behavior would be most suitable for them to change. A person who has indicated, for example, to have a positive attitude and sufficient self-efficacy expectations for a change in dietary intake will be advised to choose for a change in dietary behavior. Participants can decide whether or not they will work through this part of the program. This optional part will only be advised to individuals who indicate that they find it difficult to decide which behavior change would suit them best. Because this procedure is optional, this part is only elaborated by means of text and thus exactly the same in both versions of the intervention.

\section{Goal setting}

Goal setting is used as a method to help participants attain the desired behavior change [52]. In the program, 
participants can select a goal from a list with pre-defined options (i.e. goals) for weight, diet, and physical activity. We used a closed-ended goal setting format to prevent the formation of inadequate goals. Participants will be instructed about how to select a goal by means of written text. To further help participants set appropriate goals, they will receive extensive feedback on their behavior and weight, as mentioned before. After this feedback, participants have to decide whether they want to maintain their current weight or lose a little weight (PO 1) and how they want to achieve their weight goal, either by increasing their level of physical activity, improving dietary intake or both (PO 2). The possibility to change both behaviors is included, because several studies have shown that multiple health behavior interventions result in greater weight loss [53], have higher participation rates [54], and may be more cost-effective [53]. Individuals subsequently have to choose specific sub-behavior(s) within the chosen main behavior. Within physical activity, individuals can choose from three sub-behaviors: active transportation, physical activity in leisure time, and sports. For dietary intake a distinction is made in: dairy produce, sandwiches, food at dinner, snacks, hot and cold beverages, and alcohol. Individuals can choose a maximum of two sub-behaviors per main behavior (i.e. dietary intake and physical activity). Finally, individuals also have to decide how they want to improve the chosen subbehavior using again a pre-defined list with goals. For dietary intake, participants can choose to eat smaller portion sizes, consume a less energy-dense alternative or eat less of a certain food product. For example, an individual who chooses for snacks subsequently has to indicate whether he/she wants to eat fewer snacks, eat low energy-dense snacks or eat smaller portion sizes of snacks. For physical activity, individuals have to decide whether they want to increase the frequency or duration of activity. For example, an individual can decide to walk more times per week as well as to walk longer per time. As this whole goal setting application concerns choices and decisions individuals have to make, this part is exactly the same in both interventions and merely elaborated by means of text.

\section{Action, preparatory, and coping planning}

Research has shown that intended behavior changes are more likely to result in action when behavioral intentions are translated into concrete plans $[55,56]$. Hence, after individuals have set goals, they have to form plans for action to achieve the intended goals (PO 3). Action plans are generally made by means of the 'if-then' format [56]. The 'if' specifies when a specific action has to be performed, while the 'then' describes what specific action will be taken. In the interventions, individuals have the opportunity to make three kinds of plans: action (i.e. plans to perform the specific behavior), preparatory (i.e. plans for preparation), and coping plans (i.e. plans to deal with difficult situations). Individuals first have to formulate one action plan for every chosen sub-behavior (e.g. if I have lunch break at work, then I will eat an apple instead of a candy bar). To realize the behavior change, individuals also have the opportunity to formulate a maximum of three preparatory plans for each chosen sub-behavior (e.g. If I go to the supermarket on Monday, I will buy five apples). Both the action and preparatory plans will be formulated using an openended 'if-then' format. This format was chosen since a small pilot study among members of the target population has shown that this was the most preferred method for making action and preparatory plans. The 'if' and 'then' are predefined and individuals have to complete this sentence by filling out their own ideas and preferences. Individuals will also receive examples of good plans to help them formulate appropriate plans.

Individuals often encounter barriers that can hinder a successful behavior change [37]. Hence, a coping planning element will be introduced in the program once participants have started with the behavior change. They will be given the opportunity to make coping plans for situations in which they experience or expect difficulties to successfully perform the intended behavior. For each sub-behavior, individuals can specify a maximum of three coping plans using a closed-ended format, as the pilot study has also shown that this format was preferred for coping plans. Individuals can choose difficult situations from a pre-defined list. This list is composed based on findings from previous studies $[57,58]$ and a pilot study that was performed among members of the target population. To make a coping plan, individuals first have to choose which difficult situations they encountered or expect to encounter (e.g. a busy working day). Subsequently, they will receive feedback on how to deal with these situations followed by the opportunity to choose a coping response from a list for every chosen situation.

It should be noted that because of the large variety that can exist in plans and in order to provide clear examples of adequate plans, the planning part is carried out merely using textual messages. Hence, this part is exactly the same for the video-text and text-text intervention and there were no additional video messages.

\section{Modeling}

To target individuals' self-efficacy to carry out the desired behavior change and the corresponding plans (PO 6, 7, 8, and 10), modeling techniques are used. Narratives are used as a practical application for modeling. A narrative is a constructive format in which a sequence of events is described [59]. For each subbehavior, two narratives are created. The narratives 
follow a chronological storyline in which a role model tells how his/her behavior change is going and how he/ she deals with encountered difficulties. Each story consists of implicit tips and participants can choose whether they want to view these stories. In line with the parameters of modeling, all narratives show people who are struggling with their behavior change but eventually succeed in achieving their goal [40]. In the text-text intervention, the narratives merely consist of text, while the narratives in the video-text intervention are displayed by means of videos. In these videos an actor plays the role of a model (i.e. a person who also used the program). A different actor is used for each of the eighteen narratives in order to increase the credibility of the stories. To ensure that participants can identify themselves with the persons in the narratives, we used a variety of actors with differences in ethnic background, age, gender, educational level, and BMI. In the text-text intervention, these characteristics were described in text in the introduction of the narratives.

\section{Reinforcement and external attribution}

Besides modeling, participants' self-efficacy will also be targeted by means of reinforcement and external attribution. During the interventions, the progress toward enactment of plans and goal achievement of the participants will be evaluated. This evaluation will, for example, assess whether participants have gained, maintained, or lost weight (PO 7) as well as whether or not they have improved their physical activity level and/or dietary intake (PO 8). Participants who achieve their goals successfully will be complimented, while those who fail to achieve their goals will receive feedback in which the failure will be attributed to external factors that people cannot control in order to prevent a decrease in self-efficacy [60]. In the video-text intervention, this feedback will be provided by means of a video message, in which the progress of an individual is discussed. Simultaneously with the video message, a table is shown below the video consisting of an overview of participants' changes in body weight and dietary intake and/or physical activity during the program. In the text-text intervention, the same table will be provided, but then accompanied with text-based messages.

\section{Step 4: program development}

\section{Scope and sequence}

The program consists of six sessions. Six sessions was considered to be sufficient to go through all phases of the I-Change Model and self-regulation theories. Figure 1 provides a flowchart of the six sessions, including a brief description of their content. In session one, participants have to decide whether they want to maintain their current weight or lose a little weight and how they want to achieve this goal: improving their physical activity level, dietary intake or both (PO 1 and 2). In session two, participants have to decide which specific subbehaviors they want to change and how (PO 2), followed by the formation of action and preparatory plans (PO 3). It should be noted that the content of session one and two could have been combined into one large session, but because too much feedback may become too extensive for people to process [23], it was decided to use two separate sessions. However, at the end of session one, participants can decide to continue with session two directly. After session two, participants can start with enacting their plans and performing the desired behavior (PO 4 and 5). The remaining four sessions can be accessed in the next weeks, with at least one week between the sessions. The date for visiting the next sessions can be chosen by the participant. The focus of these sessions is on evaluating the progress toward meeting the weight and behavior change goals and enactment of the action plans and helping participants to deal with encountered or expected difficulties, for which they can make coping plans (PO 6, 7, and 8). In session four and five, participants have the possibility to change their goals and/or action plans (PO 9) and correspondingly go back to session two. In session four, five, and six, participants can further choose whether they want to view the narratives, which can help them in generating ideas for coping plans. Finally, the sixth session gives additional attention to how to maintain the behavior change on the long-term (PO 10). In total, participants can use the assigned intervention for a period of maximum three months, which is sufficient to follow all sessions. Each session lasts approximately 15 minutes, even though session two (in which current behavior is assessed) may take a bit longer to complete.

\section{Development}

The two interventions are developed using the Tailorbuilder software (OSE, the Netherlands), a program which is specifically designed to develop web-based computer-tailored interventions. To enhance participant retention and outcomes [61], the interventions are integrated into a website (http://www.gewicht-in-balans.info). This website provides information about the study, the intervention, and answers to frequently asked questions. Participants have to $\log$ in to the website in order to use their assigned intervention. Once logged in, participants also have the opportunity to read information and advices from previous sessions.

For the video-text intervention 240 videos were recorded. Because of budgetary constraints it was unfortunately not possible to convert all educational messages into videos. In all video messages an actor reads the messages aloud (i.e. the text in the text-text intervention). The video messages related to physical activity were recorded in a gym 
Session 1

Generic information about aim and content of the program

Assessment of demographic characteristics and questions concerning risk perception towards weight gain

Feedback on participants' BMI and their perceived severity and susceptibility beliefs towards weight gain

Generic information about small changes approach and body weight

Optional Information about required changes to maintain weight or lose a little weight

Goal setting: participants have to select a weight goal: weight maintenance or modest weight loss ( $5 \mathrm{~kg}$ in one year)

Short assessment of physical acitivity pattern and dietary intake

Feedback on behaviors: it will be indicated which behavior change would fit the participant best

Participants are asked if they want more information about for which behavior change they are most motivated

Optional

Assessment of motivation: attitude, self-efficacy, and social influence beliefs towards physical activity and dietary intake

Feedback on beliefs and it will be indicated which behavior change fits best with participants' motivation

Goal setting: participants have to select a weight goal: improve physical activity level,

dietary intake or both

Summary of goals and overview of session 2

Choose date of next session: continue directly, after one week or choose own date

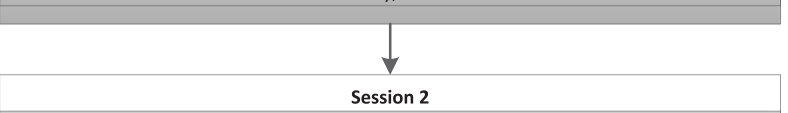

Summary of goals set in session 1 and overview of content session 2

Assessment of weight

Detailed assessment of the chosen behavior change in session 1 (physical activity, dietary intake or both)

Feedback on behavior: it will be indicated which sub-behavior changes can be made to achieve the weight goal

Goal setting: participants have to select specific sub-behavior change(s) within the chosen main behavior (i.e. dietary intake and/or physical activity)

Summary of goals and information about planning

Action planning: for each sub-behavior participants have to form 'if-then' plans by specifying when, where, and how they want to carry out the desired behavior change

Optional Preparatory planning: participants can form 'if-then' plans for preparations that have to be made before the behavior change can be carried out

Summary of goals and action and preparatory plans

Optional Information on how to best carry out action and preparatory plans

Generic information about setting rewards

Optional Setting rewards: participants can specify a future reward for when achieving their weight goal

Summary of goals and action and preparatory plans and overview of session 3.

Participants will be instructed and motivated to carry out the behavior change

Choose date of next session: after one week or choose own date

\section{Legend}

Bold $=$ video message in video-text intervention

Generic information

Assessment/questions

Tailored feedback

Act for participants

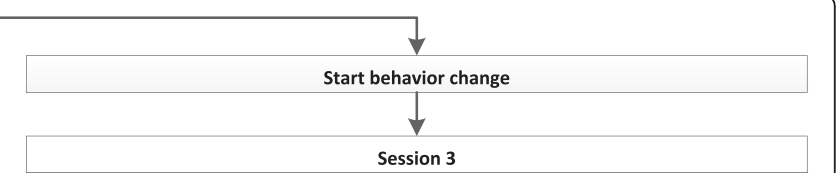

Summary of goals and action plan(s) and overview of content session 3

Assessment of weight

Assessment of the chosen sub-behavior(s) in session 2

Assessment of action plan(s) made in session 2 by asking participants when they failed to carry out their plan(s)

Feedback on sub-behavior(s): it will be indicated whether or not participants achieved their behavioral goal by comparing their current behavior with their behavior in session 2 Feedback on action plan(s): it will be indicated whether or not participants succeeded to carry out their action plan(s) successfully

Assessment of difficult situations: participants are asked if they encountered or expect to encounter situations in which they find it difficult to carry out their action plan(s)

Assessment of difficult situations: per sub-behavior participants can select a maximum of two situations in which they experienced or expected to

Optional:

coping Feedback on how to deal with the selected difficult situation(s) experience difficulties in carrying out their action plan(s)

planning Coping planning: participants can choose a coping option for how to deal with the selected difficult situation(s)

Overview of coping plan(s)

Optional Generic information on how to deal with difficult situations in general

Summary of goals and action and coping plans and overview of session 4

Choose date of next session: after one week or choose own date

\section{Session 4}

This session is idential to session 3 , only with the addition of

After the feedback on sub-behavior(s) and action plan(s): participants have the possibility to change their goals and/or action plan(s). Participants who want to change their goals and/or action plan(s) will go back to session 2 and follow the program again with their new goals and/or new action plan(s)

Optional: After the optional coping planning part, participants are asked if they want to after read/view narratives

coping
planning Narratives: per sub-behavior participants can read/view two narratives

planning

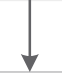

Session 5

This session is idential to session 4 , only with the addition of:

After the assessment of weight, behavior, and action plans, participants will for the first time receive feedback on their body weight during the program. It will be indicated whether or not they achieved their weight goal

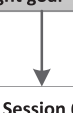

This session is idential to session 5 , only with the addition of a part related to long-term changes at the end of this last session:

Generic information about how to maintain weight (changes) on the long-term

Goal setting: participants have to set a long-term weight goal by selecting a time-frame and desired future body weight

Summary of long-term weight goal and generic information about how to maintain behavior changes on the long-term

Optional Long-term action plan(s): participants can make new action plan(s) for how to maintain the behavior change on long-term

Summary of goals and action plan(s)

Optional Recapitulation of the essentials elements of the program

Official ending of the program

Figure 1 Overview of the content of the intervention. 
and given by a professional male actor, while the messages related to dietary intake were recorded in a cooking studio using a professional female actor. These two actors had the role as an expert in the field of nutrition and physical activity. For the narratives, two actors per sub-behavior were recruited, who each had to act in three videos. These videos were recorded in a film studio that was decorated as a living room in order to increase the authenticity of the stories.

An example of the text-text intervention and the video-text intervention can be found in Additional file 2 and Additional file 3.

\section{Pretest of the interventions}

Two pretests were conducted before a definite version of the interventions was developed. First, a prototype of the text-text intervention was pretested among 25 members of the target population. Twenty individuals worked through the intervention at home, while five participants participated in a cognitive interview in the presence of a member of the project team. During this interview, participants were asked to talk out loud about the choices they made in the program and about their interpretation of the feedback, usability of the action planning tools and so on. This process yielded useful findings, for example about log in problems and unclear questions and feedback. Based on these findings, the text-text intervention was improved after which the videos were recorded. Subsequently, a pretest of the video-text intervention was conducted using the same procedure as the first pretest. This second pretest, however, focused more on the clarity of the video messages as well as the usability of the website in which the interventions are integrated. This pretest again resulted in several useful suggestions for improvement, such as speed problems during the program and difficulties with navigation through the website.

\section{Step 5: planning for program implementation}

The purpose of step five is to develop a plan for program use, including adoption, implementation, and continuation. The need to facilitate program use was, however, already taken into account from the beginning of step one by developing the interventions in such a way that will enhance their potential for being adopted, implemented, and sustained. For example, to implement the interventions no human actions are required. Individuals who want to use the intervention can go the website and register, after which they will automatically receive programmed e-mails. Furthermore, the development process of the interventions may also increase the likelihood of program use. For example, several small pilot studies among members of the target population were conducted to identify the preferences and needs of the potential users regarding the content of the intervention, the visual aspects of the website and intervention, the format of making preparatory, action, and coping plans, the recruitment materials for the evaluation study (e.g. brochure), the included difficult situations, the name of the intervention, and the actors in the video messages. During these pilot studies, we particularly investigated the opinions of individuals with a low level of education to ensure their preferences are integrated in the intervention and all intervention parts were clear to them. Moreover, by pretesting the text-text intervention and subsequently the video-text intervention, we also tried to adapt the intervention as much as possible to users' preferences and needs.

To further facilitate program use, we also composed an advisory board consisting of potential adopters and implementers, including several occupational health services. Occupational health services have the objective to provide both cure and care for working people, which is in line with the purpose of the intervention. In the Netherlands, many employees receive an annual medical screening carried out by an occupational health service [62]. During these medical screenings, employees can be handed over a brochure about the intervention. Other implementation possibilities could be the use of advertisements in mass media, social media, and newsletters in companies.

\section{Step 6: planning for program evaluation}

\section{Evaluation design and procedure}

A three-group randomized controlled trial will be conducted to evaluate the efficacy of both versions of the intervention in comparison to a waiting list control group on BMI, dietary intake, and physical activity. Participants who will be randomized to the control group can use one of the interventions after the study. Measurements will take place at baseline (T0) and at 6 (T2) and 12 months (T3) after baseline. During all measurements, participants will have to complete an online questionnaire via the study website.

A power calculation was carried out to estimate the required number of participants. In order to detect a medium sized effect $(d=0.5)$ on BMI [63], using a power of .90 , a significance level of .05 , and being able to test interaction effects between participants with a low, medium, and high level of education, about 1,000 participants need to complete the study. Taking into account a drop-out percentage of $50 \%$ between baseline and the last follow-up measurement [64], this implies that approximately 2,000 participants are needed at baseline. These will be divided over the three study arms equally. Participants are eligible for inclusion when they are at least 18 years old, have a job, and have a BMI between 18.5 and 30 . Although obese people $(B M I \geq 30)$ do not belong to the target population for the evaluation study, these individuals will be given the possibility to use the 
program. Participants who have a physical condition that may influence dietary or physical activity patterns (e.g. diabetes) are ineligible to participate. Participants will be recruited by various occupational health services during medical screenings as well as through companies without interference of the occupational health services. Brochures and messages in internal company newsletters will be used to invite people to participate in the study. Mass media advertisements and press releases will also be used to recruit participants. After registration at the study website, participants will be randomized to one of the three conditions and have the opportunity to fill out the baseline questionnaire. Participants in one of the intervention conditions have to wait two weeks before they can start using their assigned intervention. To decrease the likelihood of attrition in the evaluation study, one hundred cash prizes of $€ 100$ will be raffled among participants who complete all questionnaires [54]. Participants will also receive two reminders per intervention session and per measurement in order to improve retention in the study.

The Ethical Commission of the Open University Heerlen reviewed the study protocol and judged that the study is not within the scope of the Social Support Act (WMO) and that no approval of the medical ethics committee was required. They also indicated that there was no objection to performance of the study. The study is registered in the Dutch Trial Registry (nr. NTR3501). Participants' approval will be obtained in line with the APA informed consent ethical principles. At the beginning of the study, all eligible participants will be provided with information about the study and asked to sign an online informed consent form.

\section{Hypotheses}

The following hypotheses will be tested:

- The video-text intervention will be more effective than the text-text intervention in the prevention of weight gain (i.e. achieving weight maintenance or modest weight loss) and more appealing for individuals with low levels of education.

- The text-text intervention will be more effective than the video-text intervention in the prevention of weight gain and more appealing for individuals with high levels of education.

- Both the video-text as the text-text intervention will be more effective in the prevention of weight gain compared to the control group.

\section{Measurement instruments}

The primary outcomes of the study are self-reported body weight, BMI, physical activity, and dietary intake. These will be assessed at baseline and at 6 and 12 month follow-up. In a sample of the participants, body weight and height will also be measured objectively by various occupational health services at baseline and 12 month follow-up.

Further, several potential mediators and moderators of the intervention effects will be measured. Mediators (i.e. working mechanisms of the interventions) that will be assessed include self-regulation skills and self-efficacy and intention towards being more physically active and eating less of energy-dense food products. These will be measured at baseline and at 6 and 12 month follow-up. At baseline, various moderators will be measured as well, including demographic characteristics (e.g. gender, age, and educational level), information processing style, self-efficacy, intention, media preference, and perceived body image.

In addition, we will also conduct a process evaluation to examine potential moderating factors. This evaluation will take place directly after the last session of the intervention (T1) as well as briefly at the 6 month follow-up measurement (T2). Process measures that will be examined include action planning (i.e. the quality of action plans),

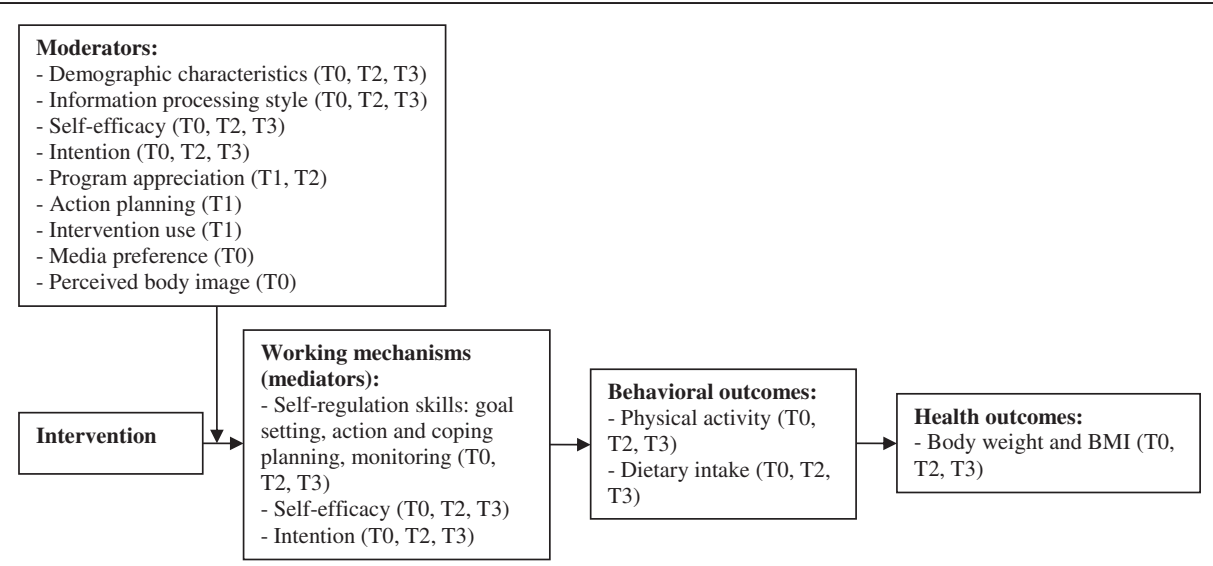

Figure 2 Overview of concepts that will be measured. Measurement times: $\mathrm{T} 0=$ baseline; $\mathrm{T} 1=$ after intervention session $6 ; \mathrm{T} 2=6$ month follow-up; T3 = 12 month follow-up. 
intervention use (e.g. number of times participants logged in and amount of information viewed), and participants' appreciation of the intervention (e.g. source, message, and channel factors). Figure 2 gives an overview of the concepts that will be measured at each time point.

\section{Discussion}

The aim of the present paper was to describe the systematic development of a text-driven and a video-driven web-based computer-tailored intervention aimed to prevent obesity among lower and higher educated Dutch adults with a healthy weight or limited overweight. Both interventions have exactly the same educational content but differ in the format in which the information is delivered. The video-text intervention provides the core messages by means of videos, while the text-text intervention is fully text-based without the use of visual elements. Both interventions follow the steps distinguished in the I-Change Model and self-regulation theories; individuals will be made aware of their health risk behavior, motivated to improve their dietary or physical activity behavior, set goals, make plans, monitor and evaluate their weight and behavior, and deal with difficult situations in order to achieve weight maintenance or modest weight loss.

The video-text intervention is specifically developed to respond to the need to develop interventions aimed at individuals with a low level of education. Despite the fact that these individuals have the highest rate of obesity $[5,6]$ and unhealthy dietary and physical activity patterns $[43,44]$, there is a lack of interventions that are appealing and effective for this high risk group. The video-text intervention may be a good way to reach these individuals, as research has shown that lower educated individuals have a preference for videos over text and evaluate video messages as more attractive and understandable than text [26,30-32]. A related advantage of the videotext intervention is the fact that videos may reduce the amount of cognition needed to process information, which will free resources to process the main message [29]. By evaluating the interventions, insight will be obtained into whether the video-text intervention is indeed more effective and appealing for lower educated individuals than the text-text intervention.

Components that may increase the efficacy of both interventions in general are the self-regulation framework and the small changes approach. Evidence indicates that these are promising ways to prevent obesity [47-49]. The fact that we used a planned approach to develop the interventions (i.e. the IM protocol) may also increase the overall efficacy [39]. Moreover, by adapting the interventions to potential users as much as possible, by integrating their preferences as indicated in several small pilot studies, the efficacy of the interventions may also be facilitated. Additionally, the interventions offer a lot of freedom. Participants can, for example, choose which goals they want to achieve and which information and advices they do or do not want to read. It has been assumed that this high sense of autonomy will enhance individuals' motivation, which in turn may increase the efficacy of the interventions [65].

To conclude, the use of the IM protocol has led to the development of two web-based computer-tailored interventions aimed to prevent obesity among Dutch adults with a healthy weight or limited overweight. By evaluating the interventions more insight will be obtained about their efficacy to prevent obesity, the efficacy of videos in web-based computer-tailored interventions, for which target group video tailoring is more effective, and by which processes effects may occur. These results can be used as input for future web-based computer-tailored interventions, specifically for those aimed at obesity prevention. If the interventions prove to be effective, an efficient obesity prevention tool that can reach large numbers of people against low costs will be available.

\section{Additional files}

Additional file 1: Table S1. Overview of performance objectives, determinants, change objectives, theoretical methods, and practical applications.

Additional file 2: Example of the text-text intervention.

Additional file 3: Example of the video-text intervention.

\section{Competing interests}

$\mathrm{HdV}$ is the scientific director of Vision2Health, a company that licenses evidence-based innovative computer-tailored health communication tools. The other authors declare that they have no competing interests.

\section{Authors' contributions}

$\mathrm{HdV}$ and $L L$ designed and wrote the original proposal. MJLW, AO, KS, LL, and $\mathrm{HdV}$ developed the obesity prevention program and execute the studies. MJLW significantly contributed to writing this paper, while $A O, K S, L L$, and $\mathrm{HdV}$ were involved in revising the manuscript critically. All authors read and approved the final manuscript.

\section{Acknowledgements}

The study was funded by ZonMw, the Netherlands Organization for Health Research and Development (grant number: 200110001).

\section{Author details}

'School for Public Health and Primary Care (CAPHRI), Maastricht University, Maastricht, The Netherlands. ${ }^{2}$ Department of Health Promotion, Maastricht University, P.O. Box 616, Maastricht 6200MD, The Netherlands. ${ }^{3}$ Department of Psychology, Open University of the Netherlands, P.O. Box 2960, Heerlen 6419AT, The Netherlands.

Received: 13 August 2013 Accepted: 8 October 2013

Published: 20 October 2013

\section{References}

1. Wang YC, McPherson K, Marsh T, Gortmaker SL, Brown M: Health and economic burden of the projected obesity trends in the USA and the UK. Lancet 2011, 378:815-825.

2. Finucane MM, Stevens GA, Cowan MJ, Danaei G, Lin JK, Paciorek CJ, Singh GM, Gutierrez HR, Lu Y, Bahalim AN, et al: National, regional, and global trends in 
body-mass index since 1980: systematic analysis of health examination surveys and epidemiological studies with 960 country-years and $9 \cdot 1$ million participants. Lancet 2011, 377:557-567.

3. World Health Organization: Obesity: preventing and managing the global epidemic. Report of a WHO consultation. Geneva: World Health Organization; 2000.

4. Bemelmans WJE, Hoogenveen RT, Visscher TLS, Verschuren WMM, Schuit AJ: Toekomstige ontwikkelingen in overgewicht - inschatting effecten op de volksgezondheid. Bilthoven: Institute for Public Health and the Environment; 2004.

5. Uiters E, Verweij A: Overgewicht: Zijn er verschillen naar sociaaleconomische status?. Bilthoven: Institute for Public Health and the Environment; 2012

6. Monteiro CA, Moura EC, Conde WL, Popkin BM: Socioeconomic status and obesity in adult populations of developing countries: a review. Bull World Health Organ 2004, 82:940-946.

7. Devaux M, Sassi F: Social inequalities in obesity and overweight in 11 OECD countries. Eur J Public Health 2011, 23:464-469.

8. Dixon JB: The effect of obesity on health outcomes. Mol Cell Endocrinol 2010, 316:104-108.

9. Swinburn BA, Caterson I, Seidell JC, James WP: Diet, nutrition and the prevention of excess weight gain and obesity. Public Health Nutr 2004 7:123-146.

10. Neovius K, Neovius M, Kark M, Rasmussen F: Association between obesity status and sick-leave in Swedish men: nationwide cohort study. Eur J Public Health 2012, 22:112-116.

11. Commission of the European Communities: A strategy for Europe on nutrition, overweight, and obesity related health issues. Brussels: European Communities; 2007.

12. Wing RR: Behavioral interventions for obesity: recognizing our progress and future challenges. Obes Res 2003, 11:3-6.

13. Gortmaker SL, Swinburn BA, Levy D, Carter R, Mabry PL, Finegood DT, Huang T, Marsh T, Moodie ML: Changing the future of obesity: science, policy, and action. Lancet 2011, 378:838-847.

14. Magnée T, Burdorf A, Brug J, Kremers SP, Oenema A, Van Assema P, Ezendam NP, Van Genugten L, Hendriksen IJ, Hopman-Rock M: Equity-specific effects of 26 Dutch obesity-related lifestyle interventions. Am J Prev Med 2013, 44:57-66.

15. Klem ML, Wing RR, McGuire MT, Seagle HM, Hill JO: A descriptive study of individuals successful at long-term maintenance of substantial weight loss. Am J Clin Nutr 1997, 66:239-246.

16. Kayman S, Bruvold W, Stern JS: Maintenance and relapse after weight loss in women: behavioral aspects. Am J Clin Nutr 1990, 52:800-807.

17. De Vries H, Brug J: Computer-tailored interventions motivating people to adopt health promoting behaviours: Introduction to a new approach. Patient Educ Couns 1999, 36:99-105.

18. Krebs P, Prochaska JO, Rossi JS: A meta-analysis of computer-tailored interventions for health behavior change. Prev Med 2010, 51:214-221.

19. Cobiac LJ, Vos T, Barendregt JJ: Cost-effectiveness of interventions to promote physical activity: a modelling study. PLoS Med 2009, 6:1-11.

20. Oenema A, Brug J, Dijkstra A, De Weerdt I, De Vries H: Efficacy and use of an internet-delivered computer-tailored lifestyle intervention, targeting saturated fat intake, physical activity and smoking cessation: a randomized controlled trial. Ann Behav Med 2008, 35:125-135.

21. Oenema A, Brug J, Lechner L: Web-based tailored nutrition education: results of a randomized controlled trial. Health Educ Res 2001, 16:647-660.

22. Brug J, Oenema A, Campbell M: Past, present, and future of computertailored nutrition education. Am J Clin Nutr 2003, 77:1028-1034.

23. Kroeze W, Werkman A, Brug J: A systematic review of randomized trials on the effectiveness of computer-tailored education on physical activity and dietary behaviors. Ann Behav Med 2006, 31:205-223.

24. Wieland LS, Falzon L, Sciamanna CN, Trudeau KJ, Brodney S, Schwartz JE, Davidson KW: Interactive computer-based interventions for weight loss or weight maintenance in overweight or obese people. Cochrane Database Syst Rev 2012, 8:1-153.

25. Paasche-Orlow MK, Parker RM, Gazmararian JA, Nielsen-Bohlman LT, Rudd RR: The prevalence of limited health literacy. J Gen Intern Med 2005, 20:175-184.

26. Wilson EA, Park DC, Curtis LM, Cameron KA, Clayman ML, Makoul G, Vom Eigen K, Wolf MS: Media and memory: the efficacy of video and print materials for promoting patient education about asthma. Patient Educ Couns 2010, 80:393-398.
27. Petty R, Cacioppo J: Communication and persuasion: central and peripheral routes to attitude change. New York: Springer-Verlag; 1986.

28. Cutler DM, Lleras-Muney A: Understanding differences in health behaviors by education. J Health Econ 2010, 29:1-28.

29. Sweller J: Cognitive load theory, learning difficulty, and instructional design. Learn Instr 1994, 4:295-312.

30. Ma Y, Hua X, Lu L, Zhang H: A generic framework of user attention model and its application in video summarization. IEEE Transactions on Multimedia 2005, 7:907-919.

31. Finn KE: Video-mediated communication. Computers, cognition, and work. Mahwah: Erlbaum; 1997

32. Calvert SL, Rideout VJ, Woolard JL, Barr RF, Strouse GA: Age, ethnicity, and socioeconomic patterns in early computer use: A National survey. Am Behav Sci 2005, 48:590-607.

33. Wang JH, Schwartz MD, Luta G, Maxwell AE, Mandelblatt JS: Intervention tailoring for Chinese American women: comparing the effects of two videos on knowledge, attitudes and intentions to obtain a mammogram. Health Educ Res 2012, 27:523-536.

34. Krawczyk A, Lau E, Perez S, Delisle V, Amsel R, Rosberger Z: How to inform: Comparing written and video education interventions to increase human papillomavirus knowledge and vaccination intentions in young adults. J Am Coll Health 2012, 60:316-322.

35. Armstrong AW, Idriss NZ, Kim RH: Effects of video-based, online education on behavioral and knowledge outcomes in sunscreen use: a randomized controlled trial. Patient Educ Couns 2011, 83:273-277.

36. Wilson EAH, Makoul G, Bojarski EA, Bailey SC, Waite KR, Rapp DN, Baker DW, Wolf MS: Comparative analysis of print and multimedia health materials: A review of the literature. Patient Educ Couns 2012, 89:7-14.

37. De Vries H, Mudde A, Leijs I, Charlton A, Vartiainen E, Buijs G, Clemente MP, Storm H, Gonzalez Navarro A, Nebot M, et al: The european smoking prevention framework approach (EFSA): an example of integral prevention. Health Educ Res 2003, 18:611-626.

38. McGuire W: Attitudes and attitude change. In Handbook of social psychology. 3rd edition. Edited by Aronson GLE. New York: Lawrence Erlbaum Associates; 1985:233-246

39. Brug J, Oenema A, Ferreira I: Theory, evidence and intervention mapping to improve behavior nutrition and physical activity interventions. Int $J$ Behav Nutr Phys Act 2005, 2:2

40. Bartholomew LK, Parcel GS, Kok G, Gottlieb NH, Fernandez ME: Planning health promotion programs: an intervention mapping approach. San Francisco: Jossey-Bass; 2011

41. Green L, Kreuter M: Health promotion planning: An educational and ecological approach. New York: McGraw-Hill; 2005.

42. Van Kreijl C, Knaap A, Van Raaij J: Our food, our health. Bilthoven: National Institute for Public Health and the Environment: 2006.

43. Giskes K, Turrell G, Van Lenthe FJ, Brug J, Mackenbach JP: A multilevel study of socio-economic inequalities in food choice behaviour and dietary intake among the Dutch population: the GLOBE study. Public Health Nutr 2006, 9:75-83.

44. Mo F, Turner M, Krewski D, Mo FD: Physical inactivity and socioeconomic status in Canadian adolescents. Int J Adolesc Med Health 2005, 17:49-56.

45. Hill J: Can a small-changes approach help address the obesity epidemic? a report of the joint task force of the american society for nutrition institute of food technologists, and international food information council. Am J Clin Nutr 2009, 89:477-484.

46. Rolls BJ, Roe LS, Meengs JS: Reductions in portion size and energy density of foods are additive and lead to sustained decreases in energy intake. Am J Clin Nutr 2006, 83:11-17.

47. De Ridder D, De Wit J: Self-regulation in health behavior. Chichester: John Wiley and Sons Ltd; 2006.

48. Schnoll R, Zimmerman BJ: Self-regulation training enhances dietary self-efficacy and dietary fiber consumption. J Am Diet Assoc 2001, 101:1006-1011.

49. Chambers JA, Swanson V: Stories of weight management: factors associated with successful and unsuccessful weight maintenance. $\mathrm{Br} J$ Health Psychol 2012, 17:223-243.

50. Abraham C, Michie S: A taxonomy of behavior change techniques used in interventions. Health Psychol 2008, 27:379-387.

51. Michie S, Abraham C, Whittington C, McAteer J, Gupta S: Effective techniques in healthy eating and physical activity interventions: a meta-regression. Health Psychol 2009, 28:690-701. 
52. Latham G, Locke E: Self-regulation through goal setting. Organ Behav Hum Decis Process 1991, 50:212-247.

53. Sweet SN, Fortier MS: Improving physical activity and dietary behaviours with single or multiple health behaviour interventions? A synthesis of meta-analyses and reviews. Int J Environ Res Public Health 2010, 7:1720-1743.

54. Robroek S, Van Lenthe F, Van Empelen P, Burdorf A: Determinants of participation in worksite health promotion programmes: a systematic review. Int J Behav Nutr Phys Act 2009, 6:26.

55. Sniehotta F, Scholzb U, Schwarzerb R: Bridging the intention-behaviour gap: planning, self-efficacy, and action control in the adoption and maintenance of physical exercise. Psychol Health 2005, 20:143-160.

56. Sheeran P, Webb TL, Gollwitzer PM: The interplay between goal intentions and implementation intentions. Pers Soc Psychol Bull 2005, 31:87-98.

57. Schulz D, Kremers S, Van Osch L, Schneider F, Van Adrichem M, De Vries H: Testing a Dutch web-based tailored lifestyle programme among adults: a study protocol. BMC Public Health 2011, 11:108.

58. Van Genugten L, Van Empelen P, Flink I, Oenema A: Systematic development of a self-regulation weight-management intervention for overweight adults. BMC Public Health 2010, 10:649.

59. Hinyard L, Kreuter M: Using narrative communication as a tool for health behavior change: a conceptual, theoretical, and empirical overview. Health Educ Behav 2007, 34:777-792.

60. Bandura A: Self-efficacy: The exercise of control. New York: Freeman; 1997.

61. Brug J, Oenema A, Kroeze W, Raat H: The internet and nutrition education: challenges and opportunities. Eur J Clin Nutr 2005, 59:130-139.

62. Institute for public health and the environment: bevolkingsonderzoeken en screeningen: Wie bieden screeningen aan? http://www.rivm.nl/Onderwerpen/ B/Bevolkingsonderzoeken_en_screeningen/Achtergrondinformatie/ Screening_de_theorie/Wie_bieden_screeningen_aan.

63. Cohen J: A power primer. Psychol Bull 1992, 112:155-159.

64. Eysenbach G: The law of attrition. J Med Internet Res 2005, 7:11.

65. Ryan RM, Deci EL: Self-determination theory and the facilitation of intrinsic motivation, social development, and well-being. Am Psychol 2000, 55:68-78.

doi:10.1186/1471-2458-13-978

Cite this article as: Walthouwer et al: Systematic development of a textdriven and a video-driven web-based computer-tailored obesity prevention intervention. BMC Public Health 2013 13:978.

\section{Submit your next manuscript to BioMed Central and take full advantage of:}

- Convenient online submission

- Thorough peer review

- No space constraints or color figure charges

- Immediate publication on acceptance

- Inclusion in PubMed, CAS, Scopus and Google Scholar

- Research which is freely available for redistribution 